\title{
Tout le monde y gagne
}

\section{Christoph Bosshard}

Dr méd., vice-président de la FMH, responsable du département DDQ

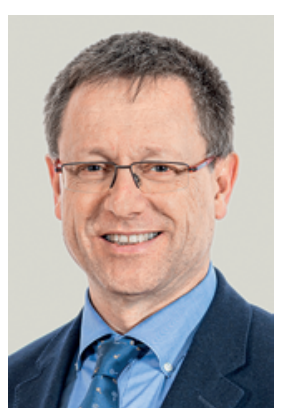

Depuis le $1^{\mathrm{er}}$ janvier 2020, l'ordonnance sur l'intégrité et la transparence dans le domaine des produits thérapeutiques (OITPTh) (www.fmh.ch/fr/prestations/droit/ oitpth.cfm) oblige les fournisseurs de prestations à répercuter sur les assurés les éventuels rabais qui ont été négociés. Mais qu'est-ce qui devrait les inciter à négocier des rabais alors qu'ils ne seront pas autorisés à les garder ensuite pour eux? La solution réside dans les dispositions de la loi sur l'assurance-maladie (LAMal). Celles-ci déterminent que les fournisseurs de prestations et/ou leurs organisations doivent répercuter sur les débiteurs de la rémunération les avantages perçus. Elles permettent aussi de prévoir, dans une convention, que les avantages visés à l'art. 56, al. 3, let. b, LAMal ne soient pas répercutés dans leur intégralité mais pour "une majeure partie» et que ceux non répercutés soient utilisés «de manière vérifiable pour améliorer la qualité du traitement». Après une phase de contrats à durée déterminée, les partenaires contractuels, à savoir la HSK, CSS, tarifsuisse et la FMH, sont parvenus à conclure une convention nationale sur la répercussion non intégrale des avantages visés à

\section{9\% au maximum des avantages perçus peuvent être utilisés pour mettre en place des mesures de qualité.}

l'art. 56, al. $3^{\text {bis }}$, LAMal. Par le biais de contrats d'adhésion, cette convention offre désormais la possibilité d'utiliser $49 \%$ au maximum des avantages perçus pour mettre en place des mesures de qualité. Car le travail de qualité coûte de l'argent. Si garantir la qualité requise fait implicitement partie intégrante de la prestation, les obligations de la documenter et de l'attester ne cessent d'augmenter. On constate partout, p. ex., que de nombreux acteurs exigent de pouvoir disposer de registres médicaux. Même si ces registres, pour autant qu'ils soient correctement tenus, génèrent de la valeur ajoutée, ils restent néanmoins un poste budgétaire dont les coûts doivent être couverts. Vous trouverez les recommandations de la FMH et les informations concernant ces registres sur le site: fmh.ch/fr/themes/ qualite-asqm/registres.cfm. Ce qui est déterminant, c'est ce que nous allons faire des données issues de ces registres. Car elles doivent être évaluées pour que nous

La moitié des avantages retourne aux assureurs et allège ainsi la charge des primes.

puissions nous en servir pour le développement de la qualité, qu'il s'agisse de fixer les thèmes des sessions de formation continue ou d'adapter les directives médicales ou la formation. La démarche qualité va aussi de pair avec l'expertise et l'expérience, ce dont disposent nos membres. Comme le financement est désormais garanti, il est alors possible d'investir le temps nécessaire.

Les payeurs de primes sont également gagnants puisque l'autre moitié des avantages retourne aux assureurs et allège ainsi le montant des primes. Le poids des primes est sur toutes les lèvres. Or, par cette convention nationale, les parties contribuent activement à réduire la part relevant de la LAMal. Pour la FMH, il est primordial d'évaluer les expériences produites par cette adaptation de loi ainsi que par les nouvelles dispositions de l'art. 58 LAMal sur la qualité et l'économicité et de l'art. 55 LAMal concernant la clause du besoin avant de décider de nouvelles révisions telles que l'introduction d'un objectif de maîtrise des coûts de l'AOs.

Tout le monde y gagne: les payeurs de primes, les fournisseurs de prestations, et surtout, le plus important, les patientes et les patients. Je remercie tous celles et ceux qui ont aidé à rendre possible cette solution! 